\title{
ZERO-HOPF PERIODIC ORBIT OF A QUADRATIC SYSTEM OF DIFFERENTIAL EQUATIONS OBTAINED FROM A THIRD-ORDER DIFFERENTIAL EQUATION
}

\author{
JAUME LLIBRE $^{1}$ AND AMMAR MAKHLOUF ${ }^{2}$
}

\begin{abstract}
We study the zero-Hopf bifurcation of the third-order differential equations

$$
x^{\prime \prime \prime}+\left(a_{1} x+a_{0}\right) x^{\prime \prime}+\left(b_{1} x+b_{0}\right) x^{\prime}+x^{2}=0,
$$

where $a_{0}, a_{1}, b_{0}$ and $b_{1}$ are real parameters. The prime denotes derivative with respect to an independent variable $t$. We also provide an estimate of the zero-Hopf periodic solution and their kind of stability. The Hopf bifurcations of these differential systems were studied in [5], here we complete these studies adding their zero-Hopf bifurcations.
\end{abstract}

\section{IntroduCtion AND STATEMENT OF THE MAIN RESUlT}

Quadratic systems of differential equations in $\mathbb{R}^{3}$ are some of the simplest systems after the linear ones and have been extensively studied in the last years. There are many examples of such systems, see for instance Lorenz system [19], the Chen system [4], the Liu system [14], the Rössler system [21], the Rikitake system [20], the Lu system [13], among several others.

One of the most interesting problems related with quadratic systems of differential equations is the study of their limit cycles, i.e. of their isolated periodic orbits in the set of all periodic orbits. It is known that every quadratic system of differential equations in $\mathbb{R}^{2}$ has finitely many limit cycles, see for instance [1], [6], [11]. For quadratic systems of differential equations in $\mathbb{R}^{n}$ with $n>2$ the scenario is very different. There are quadratic systems of differential equations for $n>2$ with infinitely many limit cycles, see for instance [7].

In [5] the authors study the nonlinear dynamics including the Hopf periodic orbits but not the zero-Hopf periodic orbits of a quadratic system of differential equations in $\mathbb{R}^{3}$ which comes from a third-order differential equation. More precisely, they analyze the third-order differential equation

$$
x^{\prime \prime \prime}+\left(a_{1} x+a_{0}\right) x^{\prime \prime}+\left(b_{1} x+b_{0}\right) x^{\prime}+c_{2} x^{2}+c_{1} x+c_{0}=0,
$$

or equivalently the first-order system of differential equations

$$
\begin{aligned}
& x^{\prime}=y, \\
& y^{\prime}=z \\
& z^{\prime}=-\left(a_{0}+a_{1} x\right) z-\left(b_{0}+b_{1} x\right) y-\left(c_{2} x^{2}+c_{1} x+c_{0}\right),
\end{aligned}
$$

2010 Mathematics Subject Classification. 37G15, 37C80, 37C30.

Key words and phrases. periodic orbit, third-order differential equation, quadratic system, averaging theory. 
where the prime denotes derivative with respect to the independent variable $t$.

The study of the different Hopf bifurcation of this system of differential equations in [5] is done in two steps, in the first the authors reduces the polynomial $c_{2} x^{2}+$ $c_{1} x+c_{0}$ to $x^{2}$, and in the second to $x(x+1)$. In both cases the authors characterize the Hopf bifurcations, i.e. when from an equilibrium point of system (1) having eigenvalues $\alpha(\varepsilon) \pm \beta(\varepsilon) i, \lambda(\varepsilon)$ it bifurcates a periodic orbit at $\varepsilon=0$ with $\alpha(0)=$ $0, \alpha^{\prime}(0) \neq 0$ and $\lambda(0) \neq 0$. The goal of this paper is to improve the study of the zero-Hopf bifurcation which exhibits in the case $x^{2}$ the system (1), i.e. from an equilibrium point of system (1) having eigenvalues $\alpha(\varepsilon) \pm \beta(\varepsilon) i, \lambda(\varepsilon)$ it bifurcates a periodic orbit at $\varepsilon=0$ with $\alpha(0)=0, \alpha^{\prime}(0) \neq 0$ and $\lambda(0)=0$. Such bifurcation was studied in [5] under the name fold-Hopf bifurcation, our two main contributions are to provided for $\varepsilon \neq 0$ sufficiently small, first an approximation of the periodic orbit which bifurcate in it, and second its kind of stability.

More precisely we first study the zero-Hopf equilibria of system

$$
\begin{aligned}
& x^{\prime}=y, \\
& y^{\prime}=z, \\
& z^{\prime}=-\left(a_{1} x+a_{0}\right) z-\left(b_{1} x+b_{0}\right) y-x^{2} .
\end{aligned}
$$

That is, the equilibrium points of a 3 -dimensional autonomous system of differential equations which have a zero eigenvalue and a pair of purely imaginary eigenvalues. In our system this zero-Hopf equilibrium is the point $(0,0,0)$ when $a_{0}=0$.

System (2) possesses the unique singular point $(x, y, z)=(0,0,0)$. In order to simplify the computations we change the parameter $b_{0}$ by a new parameter $\beta>0$ (which will be the imaginary part of the eigenvalues of the linear part of system (5) at the origin), as follows $b_{0}=\frac{1}{4}\left(\varepsilon^{2} a^{2}+\beta^{2}\right)$.

Our main result on the periodic solutions of the third-order differential equation (2) is the following.

Theorem 1. Assume that in system $(2)\left(a_{1} \beta^{2}-4\right)\left(a_{1} \beta^{2}-8\right)^{2}>0, \beta>0, b_{0}=$ $\left(a_{0}^{2}+\beta^{2}\right) / 4>0$ and $a_{0}=\varepsilon a$ where $\varepsilon$ is a small parameter, then the differential equation

$$
x^{\prime \prime \prime}+\left(a_{1} x+a_{0}\right) x^{\prime \prime}+\left(b_{1} x+\left(\varepsilon^{2} a^{2}+\beta^{2}\right) / 4\right) x^{\prime}+x^{2}=0,
$$

has the periodic solution $x(t, \varepsilon)$ such that

$$
x(0, \varepsilon)=\varepsilon \frac{4\left(\omega^{*}-r^{*}\right)}{a_{0}^{2}+\beta^{2}}+O\left(\varepsilon^{2}\right),
$$

bifurcating from a zero-Hopf equilibrium point when $\varepsilon=0$, where

$$
\left(r^{*}, \omega^{*}\right)=\left(\frac{a \beta^{4}}{\sqrt{2\left(a_{1} \beta^{2}-4\right)\left(a_{1} \beta^{2}-8\right)^{2}}},-\frac{a \beta^{4}}{4\left(a_{1} \beta^{2}-8\right)}\right) .
$$

Moreover the following statements hold.

(a) The periodic solution $x(t, \varepsilon)$ has a stable and an unstable manifolds each one formed by two cylinders if $a_{1} \beta^{2}-8<0$.

(b) The periodic solution $x(t, \varepsilon)$ is locally unstable if $a_{1} \beta^{2}-8>0$ and $a>0$.

(c) The periodic solution $x(t, \varepsilon)$ is locally stable if $a_{1} \beta^{2}-8>0$ and $a<0$. 
We note that Dias and Mello in [5] they proved the existence of such zero-Hopf bifurcation when the parameter $a_{1} \notin\left\{2 / b_{0}, 1 / b_{0}, 9 / 10 / b_{0}, 0\right\}$.

The parameter $\beta$ is taken in such a way that at the zero-Hopf bifurcation (i.e. when $\varepsilon=0$ ) the eigenvalues are $\pm \beta$ and 0 , in such a way the computations become easier. For the meaning of the other parameters see the paper [5].

Theorem 1 is proved in section 2. Its proof is based in the averaging theory for computing periodic orbits, see section 3. For others applications of the averaging theory to the study of limit cycles, see for instance $[3,15,16]$ and mainly the books $[18,22]$ where there are more than three hundred references on the averaging theory.

We shall study the system of differential equations of first order

$$
\begin{aligned}
& x^{\prime}=y, \\
& y^{\prime}=z, \\
& z^{\prime}=-\left(\varepsilon a+a_{1} x\right) z-\left(\left(\varepsilon^{2} a^{2}+\beta^{2}\right) / 4+b_{1} x\right) y-x^{2},
\end{aligned}
$$

associated to the differential equation (3). The periodic solution $u(t, \varepsilon)$ satisfying (4) of Theorem 1 written as solution of system (5) is a periodic solution which tends to the equilibrium point localized at the origin of system (5). So this periodic solution bifurcates from the origin at $\varepsilon=0$. Consequently it is a Hopf periodic solution. Moreover, since at $\varepsilon=0$ the eigenvalues of the linear part at the equilibrium $(0,0,0)$ are $\pm \beta i / 2,0$ with $\beta=2 \sqrt{b_{0}}$, we have that such periodic solution is a zero-Hopf periodic solution.

\section{Proof of Theorem 1}

First we shall see that the system of differential equations

$$
\begin{aligned}
& x^{\prime}=y, \\
& y^{\prime}=z, \\
& z^{\prime}=-\left(a_{0}+a_{1} x\right) z-\left(b_{0}+b_{1} x\right) y-x(x+1),
\end{aligned}
$$

has no zero-Hopf equilibria. Indeed, this system has two equilibria: $(0,0,0)$ and $(-1,0,0)$. The characteristic polynomials in these equilibria are $-\lambda^{3}+\left(a_{1}-a_{0}\right) \lambda^{2}+$ $\left(b_{1}-b_{0}\right) \lambda+1$ and $-\lambda^{3}-a_{0} \lambda^{2}-b_{0} \lambda-1$, respectively. Since the product of the three eigenvalues of the linear part of system (6) in these two equilibria are 1 and -1 , these equilibria never have a zero eigenvalue. So they never can be a zero-Hopf equilibrium.

The Jacobian matrix of system (5) possesses the eigenvalues $0,(-\varepsilon a \pm i \beta) / 2$. Hence when $a_{0}=\varepsilon a=0$, we have that $(0,0,0)$ is a zero-Hopf equilibrium. We shall study if from this zero-Hopf equilibrium it bifurcates or not a periodic orbit.

We shall write system (5) in such a way that the linear part at the origin will be in its real Jordan normal form. Then, doing the change of variables $(x, y, z) \rightarrow(u, v, w)$ given by

$$
\left(\begin{array}{c}
u \\
v \\
w
\end{array}\right)=\left(\begin{array}{ccc}
0 & \varepsilon \frac{a}{2} & 1 \\
0 & \frac{\beta}{2} & 0 \\
\frac{1}{4}\left(\varepsilon^{2} a^{2}+\beta^{2}\right) & \varepsilon a & 1
\end{array}\right)\left(\begin{array}{l}
x \\
y \\
z
\end{array}\right)
$$


which is equivalent to

$$
\left(\begin{array}{l}
x \\
y \\
z
\end{array}\right)=\left(\begin{array}{ccc}
-\frac{4}{\varepsilon^{2} a^{2}+\beta^{2}} & -\frac{4 \varepsilon a}{\left(\varepsilon^{2} a^{2}+\beta^{2}\right) \beta} & \frac{4}{\varepsilon^{2} a^{2}+\beta^{2}} \\
0 & \frac{2}{\beta} & 0 \\
1 & -\frac{\varepsilon a}{\beta} & 0
\end{array}\right)\left(\begin{array}{c}
u \\
v \\
w
\end{array}\right)
$$

the system of differential equations (5) becomes

(7)

$$
\begin{aligned}
u^{\prime}= & -\frac{\varepsilon a}{2} u-\frac{\beta}{2} v+\frac{4\left(a_{1}\left(\varepsilon^{2} a^{2}+\beta^{2}\right)-4\right)}{\left(\varepsilon^{2} a^{2}+\beta^{2}\right)^{2}} u^{2}+\frac{8\left(\varepsilon a\left(\varepsilon a b_{1}-4\right)+b_{1} \beta^{2}\right)}{\beta\left(\varepsilon^{2} a^{2}+\beta^{2}\right)^{2}} u v \\
& -\frac{4\left(a_{1}\left(\varepsilon^{2} a^{2}+\beta^{2}\right)-8\right)}{\left(\varepsilon^{2} a^{2}+\beta^{2}\right)^{2}} u w- \\
& -\frac{4 \varepsilon a\left(\varepsilon a\left(4+\varepsilon^{2} a^{2} a_{1}-2 \varepsilon a b_{1}\right)+\left(\varepsilon a a_{1}-2 b_{1}\right) \beta^{2}\right)}{\beta^{2}\left(\varepsilon^{2} a^{2}+\beta^{2}\right)^{2}} v^{2} \\
& +\frac{4 \varepsilon a\left(8+\varepsilon^{2} a^{2} a_{1}-2 \varepsilon a b_{1}\right)+4\left(\varepsilon a a_{1}-2 b_{1}\right) \beta^{2}}{\beta\left(\varepsilon^{2} a^{2}+\beta^{2}\right)^{2}} v w-\frac{16}{\left(\varepsilon^{2} a^{2}+\beta^{2}\right)^{2}} w^{2}, \\
v^{\prime}= & \frac{\beta}{2} u-\frac{\varepsilon a}{2} v, \\
w^{\prime}= & \frac{4\left(a_{1}\left(\varepsilon^{2} a^{2}+\beta^{2}\right)-4\right)}{\left(\varepsilon^{2} a^{2}+\beta^{2}\right)^{2}} u^{2}+\frac{8\left(\varepsilon a\left(-4+\varepsilon a b_{1}\right)+b_{1} \beta^{2}\right)}{\beta\left(\varepsilon^{2} a^{2}+\beta^{2}\right)^{2}} u v \\
& -\frac{4\left(a_{1}\left(\varepsilon^{2} a^{2}+\beta^{2}\right)-8\right)}{\left(\varepsilon^{2} a^{2}+\beta^{2}\right)^{2}} u w-\frac{4 \varepsilon a\left(\varepsilon a\left(4+\varepsilon^{2} a^{2} a_{1}-2 \varepsilon a b_{1}\right)+\left(\varepsilon a a_{1}-2 b_{1}\right) \beta^{2}\right)}{\beta^{2}\left(\varepsilon^{2} a^{2}+\beta^{2}\right)^{2}} v^{2} \\
& +\frac{4 \varepsilon a\left(8+\varepsilon^{2} a^{2} a_{1}-2 \varepsilon a b_{1}\right)+4\left(\varepsilon a a_{1}-2 b_{1}\right) \beta^{2}}{\beta\left(\varepsilon^{2} a^{2}+\beta^{2}\right)^{2}} v w-\frac{16}{\left(\varepsilon^{2} a^{2}+\beta^{2}\right)^{2}} w^{2} .
\end{aligned}
$$

Note that the linear part of the system of differential equations (7) at the origin is in its real normal form of Jordan. Doing the rescaling $(u, v, w)=(\varepsilon U, \varepsilon V, \varepsilon W)$, and system (7) becomes

$$
\begin{aligned}
U^{\prime}= & -\frac{\beta}{2} V-\varepsilon \frac{a}{2} U-\varepsilon \frac{1}{2 \beta^{4}}\left(-8 a_{1} U^{2} \beta^{2}+8 a_{1} U W \beta^{2}-16 b_{1} U V \beta+\right. \\
& \left.16 b_{1} V W \beta+32 U^{2}+32 W^{2}-64 U W\right)+O\left(\varepsilon^{2}\right), \\
V^{\prime}= & \frac{\beta}{2} U-\varepsilon \frac{a}{2} V, \\
W^{\prime}= & \varepsilon \frac{4(U-V)\left(\left(a_{1} \beta^{2}-4\right) U+2 \beta b_{1} V+4 W\right)}{\beta^{4}}+O\left(\varepsilon^{2}\right) .
\end{aligned}
$$


Consider the polar coordinates $U=r \cos \theta, V=r \sin \theta$ and $W=\omega$. The system of differential equations (8) in these coordinates writes

$$
\begin{aligned}
\frac{d r}{d t}= & \varepsilon \frac{1}{2 \beta^{4}}\left(8 r^{2}\left(a_{1} \beta^{2}-4\right) \cos ^{3} \theta-a r \beta^{4} \sin ^{2} \theta\right. \\
& -16 \omega \cos \theta\left(2 \omega+b_{1} r \beta \sin \theta\right) \\
& \left.+r \cos ^{2} \theta\left(64 \omega-8 a_{1} \beta^{2} \omega-a \beta^{4}+16 b_{1} \beta r \sin \theta\right)\right)+O\left(\varepsilon^{2}\right), \\
\frac{d \theta}{d t}= & \frac{\beta}{2}+\varepsilon \frac{4}{\beta^{4} r}(\omega-r \cos \theta) \sin \theta\left(4 \omega+r\left(a_{1} \beta^{2}-4\right) \cos \theta+2 b_{1} \beta r \sin \theta\right) \\
& +O\left(\varepsilon^{2}\right), \\
\frac{d \omega}{d t}= & -\varepsilon \frac{4}{\beta^{4}}(\omega-r \cos \theta)\left(4 \omega+r\left(a_{1} \beta^{2}-4\right) \cos \theta+2 b_{1} \beta r \sin \theta\right)+O\left(\varepsilon^{2}\right) .
\end{aligned}
$$

Therefore the solutions of system (9) in the region $\dot{\theta}>0$ can be studied analyzing the solution of the system of differential equations

(10)

$$
\begin{aligned}
\frac{d r}{d \theta}= & \varepsilon \frac{1}{\beta^{5}}\left(8 r^{2}\left(a_{1} \beta^{2}-4\right) \cos ^{3} \theta-a r \beta^{4} \sin ^{2} \theta-16 \omega \cos \theta\left(2 \omega+b_{1} r \beta \sin \theta\right)\right. \\
& \left.+r \cos ^{2} \theta\left(64 \omega-8 a_{1} \beta^{2} \omega-a \beta^{4}+16 b_{1} \beta r \sin \theta\right)\right)+O\left(\varepsilon^{2}\right), \\
\frac{d \omega}{d \theta}= & -\varepsilon \frac{8}{\beta^{5}}(\omega-r \cos \theta)\left(4 \omega+r\left(a_{1} \beta^{2}-4\right) \cos \theta+2 b_{1} \beta r \sin \theta\right)+O\left(\varepsilon^{2}\right) .
\end{aligned}
$$

For applying the averaging theorem we compute the averaged system (15). We obtain

$$
\begin{aligned}
& \frac{d r}{d \theta}=-\varepsilon \frac{1}{\beta^{5}} r\left(a \beta^{4}+4 \omega\left(a_{1} \beta^{2}-8\right)\right)=\varepsilon g_{1}(r, \omega), \\
& \frac{d \omega}{d \theta}=\varepsilon \frac{4}{\beta^{5}}\left(-8 \omega^{2}+r^{2}\left(a_{1} \beta^{2}-4\right)\right)=\varepsilon g_{2}(r, \omega) .
\end{aligned}
$$

The unique equilibrium point of system (11) with $r>0$ is

$$
\left(r^{*}, \omega^{*}\right)=\left(\frac{a \beta^{4}}{\sqrt{2\left(a_{1} \beta^{2}-8\right)^{2}\left(a_{1} \beta^{2}-4\right)}},-\frac{a \beta^{4}}{4\left(a_{1} \beta^{2}-8\right)}\right),
$$

recall that by assumptions $\left(a_{1} \beta^{2}-8\right)^{2}\left(a_{1} \beta^{2}-4\right)>0$.

The zero $\left(r^{*}, \omega^{*}\right)$ of system

$$
\left(\begin{array}{l}
g_{1}(r, \omega) \\
g_{2}(r, \omega)
\end{array}\right)=\left(\begin{array}{l}
0 \\
0
\end{array}\right)
$$

with respect to the variables $r$ and $\omega$ provide a periodic solution of system $(10)$ with $\varepsilon \neq 0$ sufficiently small if it is simple, i.e. if

$$
\operatorname{det}\left(\left.\frac{\partial\left(g_{1}, g_{2}\right)}{\partial(r, \omega)}\right|_{(r, \omega)=\left(r^{*}, \omega^{*}\right)}\right)=\frac{16 a^{2}}{\beta^{2}\left(a_{1} \beta^{2}-8\right)} \neq 0,
$$

which is the case by assumptions. By Theorem 2, system (10) has a periodic solution $(r(\theta, \varepsilon), \omega(\theta, \varepsilon))$ such that $(r(0, \varepsilon), \omega(0, \varepsilon))=\left(r^{*}, \omega^{*}\right)+O(\varepsilon)$.

This periodic solution in the coordinates $(r, \theta, \omega)$ of system $(9)$ writes $(r(t, \varepsilon), \theta(t, \varepsilon)$, $\omega(t, \varepsilon))$ and satisfies $(r(0, \varepsilon), \theta(0, \varepsilon), \omega(0, \varepsilon))=\left(r^{*}, 0, \omega^{*}\right)+O(\varepsilon)$. 
Now the periodic solution written in the coordinates $(U, V, W)$ of system (8) becomes $(U(t, \varepsilon), V(t, \varepsilon), W(t, \varepsilon))$ and satisfies $(U(0, \varepsilon), V(0, \varepsilon), W(0, \varepsilon))=\left(r^{*}, 0, \omega^{*}\right)+$ $O(\varepsilon)$.

Again this last periodic solution written in the variables $(u, v, w)$ of system $(7)$ is $(u(t, \varepsilon), v(t, \varepsilon), w(t, \varepsilon))$ satisfying $(u(0, \varepsilon), v(0, \varepsilon), w(0, \varepsilon))=\varepsilon\left(r^{*}, 0, \omega^{*}\right)+O\left(\varepsilon^{2}\right)$.

Finally this periodic solution in the variable $(x, y, z)$ of system (5) becomes $(x(t, \varepsilon), y(t, \varepsilon), z(t, \varepsilon))$ satisfying

$$
(x(0, \varepsilon), y(0, \varepsilon), z(0, \varepsilon))=\varepsilon\left(\frac{4\left(\omega^{*}-r^{*}\right)}{\varepsilon^{2} a^{2}+\beta^{2}}, 0, r^{*}\right)+O\left(\varepsilon^{2}\right) .
$$

Hence, this periodic solution in the differential equation $(3)$ is $x(t, \varepsilon)$ satisfying $x(0, \varepsilon)=\varepsilon \frac{4}{\varepsilon^{2} a^{2}+\beta^{2}}\left(\omega^{*}-r^{*}\right)+O\left(\varepsilon^{2}\right)$.

In order to complete the proof of Theorem 1, we must prove the statements (a) $-(\mathrm{d})$.

The eigenvalues of the matrix

$$
\left(\left.\frac{\partial\left(g_{1}, g_{2}\right)}{\partial(r, \omega)}\right|_{(r, \omega)=\left(r^{*}, \omega^{*}\right)}\right)
$$

are

$$
\frac{8 a-4 a \sqrt{12-a_{1} \beta^{2}}}{\beta\left(a_{1} \beta^{2}-8\right)} \text { and } \frac{8 a+4 a \sqrt{12-a_{1} \beta^{2}}}{\beta\left(a_{1} \beta^{2}-8\right)} .
$$

So the trace of the matrix (13) is

$$
\frac{16 a}{\beta\left(a_{1} \beta^{2}-8\right)}
$$

From the expression of the determinant of the matrix (13) given in (12) it follows that the equilibrium point $\left(r^{*}, \omega^{*}\right)$ of the averaged system (11) is a saddle when $a_{1} \beta^{2}-8<0$. Therefore by statement (c) of Theorem 2 the corresponding periodic orbit of system (2) has two stable and two unstable manifolds formed by cylinders, and statement (a) of Theorem 1 is proved.

If $a_{1} \beta^{2}-8>0$ and $a>0$, then the trace of the matrix (13) is positive. So some of the two eigenvalues of this matrix has a positive real part. Hence the equilibrium point $\left(r^{*}, \omega^{*}\right)$ of the averaged system (11) is unstable, and consequently by statement (c) of Theorem 2 the corresponding periodic orbit of system (2) is unstable. This proves statement (b) of Theorem 1.

If $a_{1} \beta^{2}-8>0$ and $a<0$, then the trace of the matrix (13) is negative, and both eigenvalues have negative real part. Therefore the equilibrium point $\left(r^{*}, \omega^{*}\right)$ of the averaged system (11) is stable, and consequently by statement (c) of Theorem 2 the corresponding periodic orbit of system (2) is stable. This proves statement (c) of Theorem 1. This completes the proof of Theorem 1. 


\section{Averaging theOry OF FIRSt ORDER}

We work with the two initial value problems

$$
\dot{\mathbf{x}}=\varepsilon F_{1}(t, \mathbf{x})+\varepsilon^{2} F_{2}(t, \mathbf{x}, \varepsilon), \quad \mathbf{x}(0)=\mathbf{x}_{0},
$$

and

$$
\dot{\mathbf{y}}=\varepsilon g(\mathbf{y}), \quad \mathbf{y}(0)=\mathbf{x}_{0},
$$

with $\mathbf{x}, \mathbf{y}$ and $\mathbf{x}_{0}$ in some open $\Omega$ of $\mathbb{R}^{n}, t \in[0, \infty), \varepsilon \in\left(0, \varepsilon_{0}\right]$. Let $\mathbf{F}_{\mathbf{1}}$ and $\mathbf{F}_{\mathbf{2}}$ be periodic functions of period $\mathrm{T}$ in the variable $\mathrm{t}$, and we define

$$
g(\mathbf{y})=\frac{1}{T} \int_{0}^{T} F_{1}(t, \mathbf{y}) d t .
$$

Theorem 2. By hypotheses the functions $F_{1}, D_{\mathbf{x}} F_{1}, D_{\mathbf{x x}} F_{1}$ and $D_{\mathbf{x}} F_{2}$ are continuous, bounded by a constant independent of $\varepsilon$ in $[0, \infty) \times \Omega \times\left(0, \varepsilon_{0}\right]$, and that $y(t) \in \Omega$ for $t \in[0,1 / \varepsilon]$. Then the next statements are satisfied.

(a) For $t \in[0,1 / \varepsilon]$ it follows that $\mathbf{x}(t)-\mathbf{y}(t)=O(\varepsilon)$ as $\varepsilon \rightarrow 0$.

(b) If $p \neq 0$ is an equilibrium point of system (15) and $\operatorname{det} D_{\mathbf{y}} g(p) \neq 0$, then there is a periodic solution $\phi(t, \varepsilon)$ of period $T$ for system (14) such that $\phi(0, \varepsilon)-p=O(\varepsilon)$ as $\varepsilon \rightarrow 0$.

(c) The kind of stability of the periodic solution $\phi(t, \varepsilon)$ is the same than the stability of the equilibrium point.

The notation $D_{\mathbf{x}} g$ means the Jacobian matrix of $g$ with respect to the components of $\mathbf{x}$, and $D_{\mathbf{x x}} g$ means the Hessian matrix of $g$.

For a proof of Theorem 2 see [23].

The averaging theory at any order in dimension one is developed in [8], and in arbitrary dimension in [17]. Moreover it has been proved the equivalence of the averaging method at first order in dimension two with the Abelian integral method see [9], and recently the equivalence between the averaging method and the Melnikov function method at any order in $[2,10]$.

\section{ACKNowledgements}

We thank to the reviewers their comments which help us to improve the presentation of this paper.

The first author is partially supported by a FEDER-MINECO grant MTM201677278-P, a MINECO grant MTM2013-40998-P, and an AGAUR grant number 2014SGR-568.

\section{REFERENCES}

[1] R. BAmon, Quadratic vector fields in the plane have a finite number of limit cycles, Inst. Hautes Etudes Sci. Publ. Mat. 64 (1986), 111-142.

[2] A. BuICA, On the Equivalence of the Melnikov Functions Method and the Averaging Method, Qual. Theory Dyn. Syst. in press (DOI: 10.1007/s12346-016-0216-x).

[3] C.A. Buzzi, J. Llibre, J.C. Medrado and J. Torregrosa, Bifurcation of limit cycles from a center in $\mathbb{R}^{4}$ in resonance 1: N, Dyn. Sys. : An Int. Journal 24 (2009), 123-137. 
[4] G. Chen and T. Ueta, Yet another chaotic attractor, Int. J. Bifur. Chaos 9 (1999), 14651466 .

[5] F.S. Dias And L.F. Mello, Nonlinear analysis of a quadratic system obtained from a scalar third order differential equation, Electron. J. Diff. Equ. 161 (2010), 1-25.

[6] J. ECALLE, Introduction aux fonctions analysables et preuve constructive de la conjecture de Dulac (French), Hermann, Paris, 1992.

[7] A. Ferragut, J. Llibre And C. Pantazi, Polynomial vector fields in $\mathbb{R}^{3}$ with infinitely many limit cycles, Int. J .Bifurcation and Chaos 23 (2013), 1350029-10 pp.

[8] J. Giné, M. Grau And J. Llibre, Averaging theory at any order for computing periodic orbits, Phys. D 250 (2013), 58-65.

[9] J. GinÉ AND J. LliBRE, Limit cycles of cubic polynomial vector fields via the averaging theory, Nonlinear Anal. 66 (2007), 1707-1721.

[10] M. Han, V.G. Romanovski and X. Zhang, Equivalence of the Melnikov function method and the averaging method, Qual. Theory Dyn. Syst. 15 (2016), 471-479.

[11] Y. Ilyashenko, Finiteness Theorems for limit cycles, American Mathematical Society, Providence, RI, 1993

[12] Y.A. Kuznetsov, Elements of Applied Bifurcation Theory, Springer-Verlag, 3rd edition, 2004.

[13] J. Lu And G. Chen, A new chaotic attractor coined, Int. J. Bifurcation and Chaos 12 (2002), 659-661.

[14] C. Liv, T. Liu, L. Liu and K. Liu, A new chaotic, Chaos, Solitons and Fractals 22 (2004), $1031-1038$

[15] J. Llibre ANd A. Makhlouf, Periodic solutions for a class of non-autonomous Newton differential equations, Differ Equ Dyn Syst, DOI 10.1007/s12591-016-0333-7, 2017.

[16] J. Llibre, A.C. Mereu And M.A. Teixeira, Limit cycles of resonant four-dimensional polynomial system, Dyn. Sys. : An Int. Journal 25 (2010), 145-158.

[17] J. Llibre, D.D. Novaes and M.A. Teixeira, Higher order averaging theory for finding periodic solutions via Brouwer degree, Nonlinearity 27 (2014), 563-583.

[18] J. Llibre, R. Moeckel and C. Simó, Central configurations, periodic orbits and Hamiltonian systems, Advances Courses in Math., CRM Barcelona, Birhauser, 2015.

[19] E.N. LoRenz, Deterministic nonperiodic flow, J. Atmos. Sci. 20 (1963), 130-141.

[20] T. Rikitake, Oscillations of a system of disk dynamos, Proc. R. Cambridge Philos. Soc. 54 (1958), 89-105

[21] E. Rössler, An equation for continuous chaos, Phys. Lett. A, 57 (1976), 397-398.

[22] J.A. Sanders F. Verhulst And J. Murdock, Averaging Methods in Nonlinear Dynamical Systems (second edition), Applied Mathematical Sciences 59, Springer, New York, 2007.

[23] F. Verhulst, Nonlinear Differential Equations and Dynamical Systems, Universitext, Springer, New York, 1996.

1 Departament de Matematiques, Universitat Autònoma de Barcelona, 08193 Bellaterra, Barcelona, Catalonia, Spain

E-mail address: jllibre@mat.uab.cat

2 Department of Mathematics, University of Annaba, Elhadjar 23 Annaba, Algeria

E-mail address: makhloufamar@yahoo.fr 